\title{
Motor neurone disease: the nature of the pathogenic mechanism
}

\author{
D. M. A. MANN AND P. O. YATES
}

From the Department of Neuropathology, Manchester University, Manchester

SYNOPSIS Evidence is presented which indicates that the initial site of action of the pathogen of motor neurone disease (MND) is the nucleus, and its prime effect is to cause the progressive inhibition of DNA directed mRNA synthesis by the slow condensation of chromatin from a metabolically active diffuse form to an inactive form. The other pathological changes observed follow as part of a nonspecific cell atrophy which leads eventually to dysfunction, death, and disappearance of cells.

In 1869, Charcot and Joffroy, in the first full description of motor neurone disease (MND), described it as a slowly progressive disease of unknown aetiology beginning in adult life. Since that time, very little advance has been made in understanding either the aetiology or the pathogenesis of this distressing condition with its relentless course lasting usually between three months and three years.

Pathologically, there is seen a loss of motor cells from anterior horns of the spinal cord, motor nuclei of brain-stem and motor cortex, and degenerative changes in remaining cells of these areas. The cell body is usually shrunken and may contain excessive amounts of lipofuscin. Nissl granules (RNA) are often lost and the remainder conglomerated into large masses which lie near the cell membrane. Nuclear changes are common. The nucleolus is shrunken and loses its basophilia. Nuclear chromatin, which is relatively inconspicuous in normal cells, is prominent and in severely diseased neurones the nucleus is usually shrunken and heterochromatic, while even in mildly affected cells some chromatin clumping is seen.

A primary disorder of neuronal metabolism has come increasingly into favour as a pathogenic mechanism for the disease but the real nature of this has remained far from clear. The genetic transmission which is seen in some cases (Kurland and Mulder, 1954, 1955) is in keeping with such a metabolic lesion. So far, studies to determine the nature of the MND pathogen have $\stackrel{0}{\omega}$ fallen into three categories:

1. The induction of the neurological syndrome $\overrightarrow{ } \vec{\circ}$ in inoculated animals by the transmission of $\mathrm{a}$ 웅 (so-called) slow virus from tissue homogenates of $\stackrel{\mathbb{Q}}{\stackrel{\Upsilon}{?}}$ 을 MND patients. In 1963 Zil'ber and colleagues $\stackrel{\mathbb{\Omega}}{\circ}$ reported that they had successfully transmitted $\widetilde{D}$ MND to rhesus monkeys, after inoculation with 8 $\frac{\mathbb{D}}{\mathbb{D}}$ brain extracts from patients who had died of 3 MND, which after a latent period developed the MND syndrome. On the basis of these results, $\stackrel{?}{\overrightarrow{0}}$ Gibbs and Gadjusek (1968) carried out a series of experiments using their own and Zil'ber's tissue extracts, but have so far been unable to confirm the original report of Zil'ber (1963).

2. The induction of a neurological syndrome in animals by neurotoxins has been used in an attempt to create a metabolic analogy to MND. The neurotoxins so far used are the substituted fluoropyrimidines and antibiotics such as actinomycin D (Koenig, 1968b), organophosphorous and organomercuric compounds, and isoniazid (Cavanagh, 1968). Although the use of such neurotoxins may act as a model for human diseases, the results must be interpreted with caution and analogies not pushed too far.

3. Histochemical studies (Friede and Dejong, 1964; Robinson, 1967; Friede, 1968; Koenig, 1968a) show losses of oxidative type enzyme activities paralleling the loss of large motor neurones, whereas minimal changes are seen in hydrolytic enzymes. In the degenerating tracts 


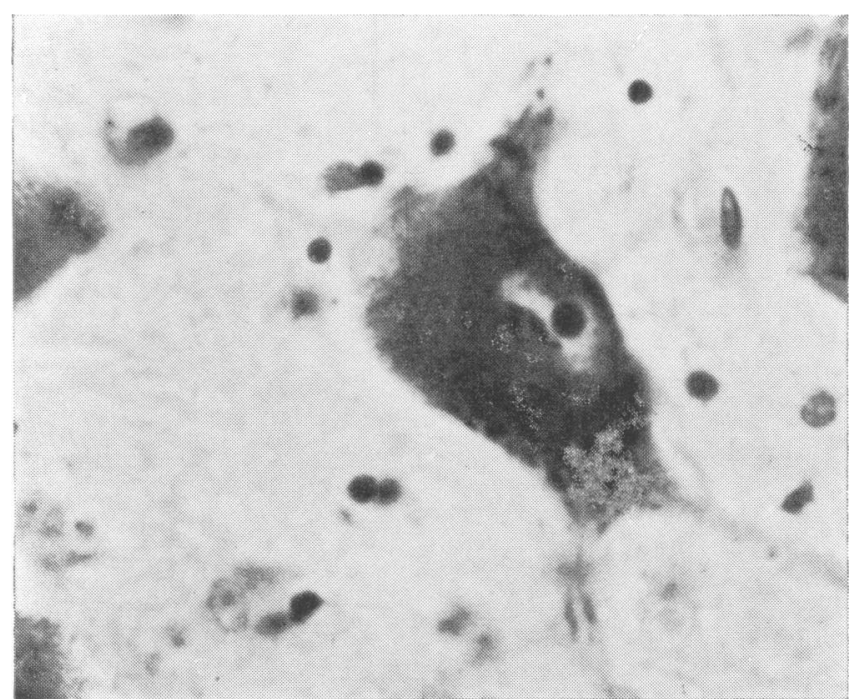

FIG. 1. Cell of motor nucleus of the trigeminal nerve of MND case A, showing nuclear shrinkage but no loss of nucleolar or cytoplasmic RNA staining. Thionine, $\times 850$.

increases in oxidative enzyme activity are seen, due to an increased activity of reactive astrocytes (Engel, 1968; Friede, 1968). There is seen a loss of acetyl cholinesterase activity, consistent with axonal degeneration (Friede, 1968). Acid phosphatase is generally increased, indicative of an increase in lysosomal activity in response to tissue destruction.

From these studies no specific biochemical abnormality was noted, and no primary defect of any particular enzyme has been found. The atrophic neurone is seen as a cell of diminished biosynthetic activity and augmented catabolic activity. The only definite changes to emerge from these studies are those due to the reactive astrocytosis and are considered secondary.

A criticism of most histochemical studiesand perhaps a cause of their failure to determine a specific biochemical lesion-lies in the fact that all were based on material from longstanding cases, obtained at necropsy, in which any initial biochemical changes are hardly likely to remain in affected neurones.

In our study, observations were made on a case of motor neurone disease (case A) which was of only four months' duration and was prematurely terminated as a result of accidental death, and compared with those made on a typical long-standing case of motor neurone disease (case B) and a normal control free from neurological illness (case $\mathrm{C}$ ). In the first case it was thought possible that the disease might not yet have progressed to such an extent that all affected cells would be in an advanced atrophic condition, and initial pathological changes might be still present in some cells.

A brief summary of the clinical history and neuropathology of both MND cases now follows.

\section{CASE A}

CLINICAL HISTORY At the age of 77 years the patient developed a weakness of the left arm and hand which rapidly spread to involve the right arm and both legs. On examination he was found to have wasting and weakness of the muscles of the left arm, and widespread fasciculation of all muscles of the arms and legs. Power was markedly diminished in the left arm and shoulder and slightly less so in the right arm and flexors of the hips and knees. There was wasting of the small muscles of both hands (left greater than right), and the patient had difficulty in using the fingers due to 'stiffness'. Reflexes were generally depressed or absent. Right facial weakness was seen, but otherwise the cranial nerves showed no abnormality.

Two months later the limb muscles had so weakened that the patient had severe difficulty in walking. Fasciculation of the tongue was present, but no further cranial nerve involvement was seen. Electromyographic studies were consistent with a diagnosis 


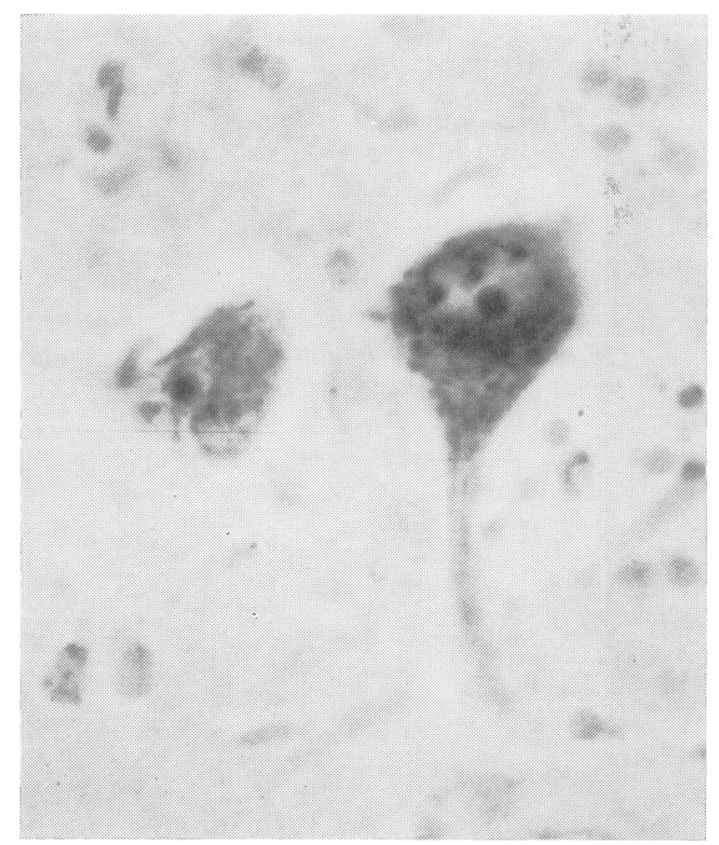

FIG. 2. Cell of motor nucleus of the trigeminal nerve of MND case A, showing shrinkage of cell body and nucleus. There is loss of cytoplasmic RNA and the nucleolus shows reduced staining. Thionine, $\times 850$.

of motor neurone disease. Shortly afterwards the patient was admitted to hospital after an accident. He did not respond to treatment and died of bronchopneumonia without regaining consciousness.

\section{CASE B}

The patient was first examined at the age of 42 years with an 18 month history of tremor of the left arm and leg. Since then she had shown features of Parkinsonism more severely affecting the left side, and developed a tendency to drag her right foot. By two years before her death she had developed limb weakness and difficulty in swallowing. She was seen to have bilateral facial weakness and bulbar palsy, which caused much difficulty in speaking and swallowing. There was gross wasting of the neck muscles, such that she was unable to extend her head. There was marked weakness, wasting, and fasciculation of all limb muscles, especially in the arms. No abnormality of sensation was noted. Electromyographic studies revealed widespread denervation in all limbs. She was admitted to hospital with severe difficulty in eating, speaking, and coughing; she continued to deteriorate until her death at the age of 52 years.

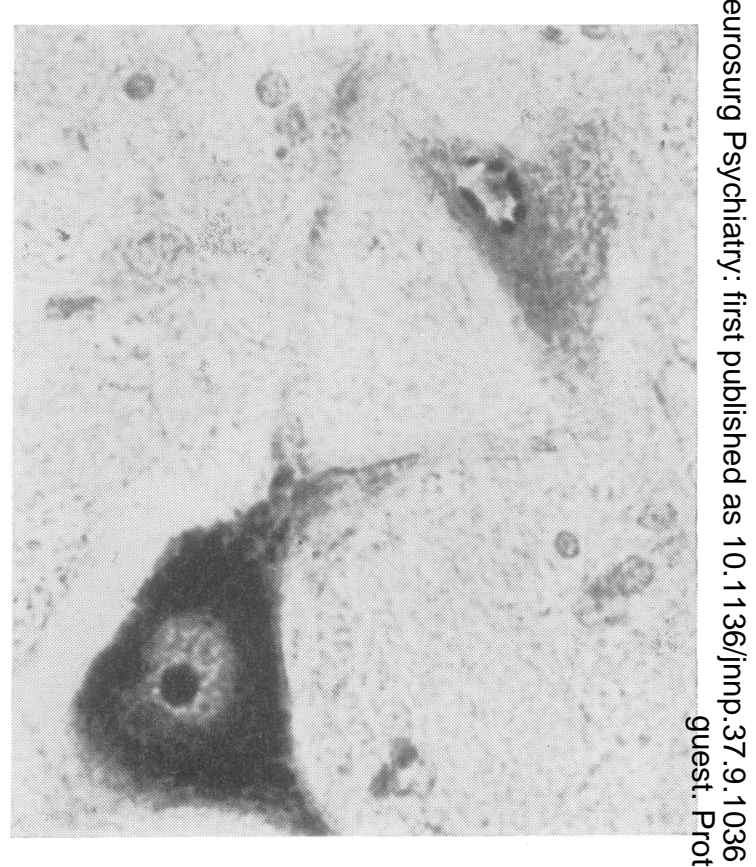

FIG. 3. Cells of motor nucleus of the trigeminal nerise 을 of MND case $A$; one shows complete loss of cyt plasmic $R N A$, with further reduction in nucleold $\mathbb{D}$ staining. The DNA is represented by a few large heterochromatic clumps. Thionine, $\times 850$.

PATHOLOGICAL FINDINGS In both cases all findingS $\overrightarrow{0}$ were consistent with the diagnosis of motor neurone $\perp$ disease. The most severe changes were seen in the anterior horn cells of the spinal cord (especially the ventromedial cell column), Betz cells of cerebral cortex, and cells of the hypoglossal nucleus. These cells were in an advanced state of degeneration; many of them were completely shrunken, showing a great loss of Nissl substance. The nucleus was severely shrunken and in many cells the chromatin was represented by a few large heterochromatic granules. The nucleolus was either absent or severely atrophied.

Better preserved were the motor neurones of the cranial nerve nuclei; changes in the cells of the VI, VII, VIII, and XII nuclei being more severe than in the III, IV, and V nuclei. In the oculomotor and trigeminal motor nerve nuclei of both cases, cells of normal appearance were seen scattered among 옹 obviously diseased neurones. This was especially so in case A. Diseased neurones showed a variety of changes. In early stages the cell body was rounded, N swollen, and showed a loss of dendrites. The nucleus was sometimes slightly shrunken, in which case the 


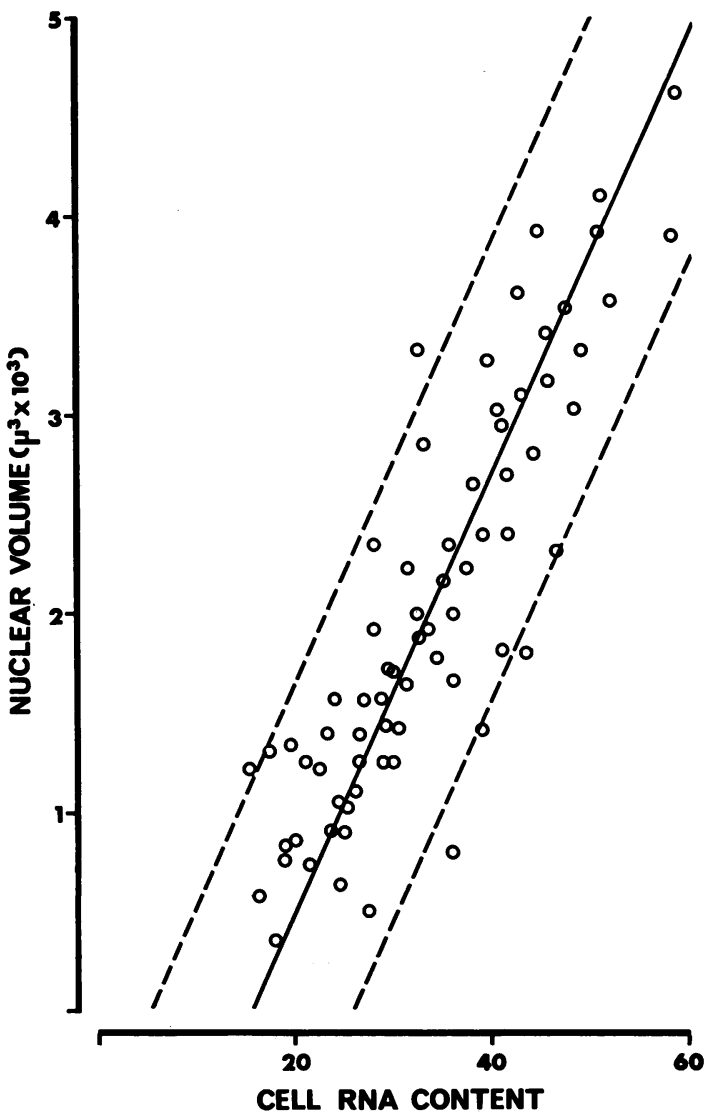

FIG. 4. Graph of nuclear volume plotted against cytoplasmic (cell) RNA content for anterior horn cells of the control case.

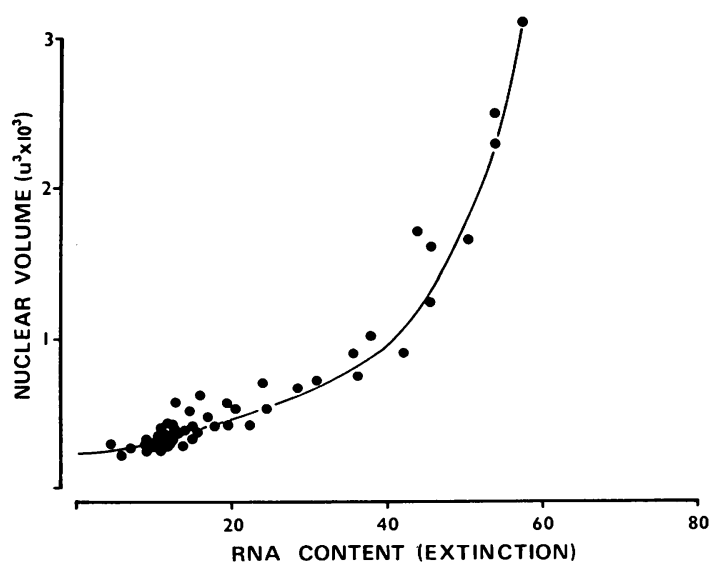

FIG. 5. Graph of nuclear volume plotted against cytoplasmic RNA content for anterior horn cells of MND case $A$. chromatin was often clumped into heterochromatic granules; the nucleolus was normally stained. Nissl granules were either unaltered or fine and powdery centrally, while larger granules were peripherally sited (Fig. 1).

Later stages showed cell shrinkage and loss of Nissl granules, the remainder being clumped into large masses. The nucleolus showed reduced affinity for basic dyes, and the nuclear chromatin was shrunken (Fig. 2). Later still there was further cell shrinkage with the remaining cytoplasm virtually devoid of RNA staining. The DNA was represented by a few heterochromatic clumps and the nucleolus was either degenerate or absent (Fig. 3). Finally, the remaining cytoplasm had lost all nucleic acids and contained only the hydrolytically resistant lipofuscin granules.

Such a mixed population containing normal and diseased cells was therefore ideal for the following quantitative study.

\section{METHODS}

Nervous tissue was obtained at necropsy from both MND and control cases within 12 hours of death. The results of an autolysis experiment (Mann, 1972) showed that during a period of 11 hours of refrigerated storage after death, nervous tissue lost the majority of low molecular weight RNA species (transfer and messenger RNA which could not therefore be studied) but no cytoplasmic or ribosomal RNA (Nissl substance) or DNA).

Blocks of tissue were fixed in $10 \%$ neutral formalin, embedded in paraffin wax, and sections were cut at $16 \mu \mathrm{m}$ thickness. Sections were stained for DNA and RNA using the Feulgen and Azure B (Shea, 1970) methods respectively. Measurements of both DNA and RNA contents were made using the Leitz MPV microspectrophotometer. For estimations of DNA content, the two wavelength method of Ornstein (1952) and Patau (1952) was employed at wavelengths of 500 and $552 \mathrm{~m} \mu \mathrm{m}$ using a $\times 100$ oil immersion objective; calculations of DNA content were made using the set of tables compiled by Mendelsohn (1958). RNA content was estimated as the percentage absorption of light at $560 \mathrm{~m} \mu \mathrm{m}$, using a $\times 40$ objective. The $100 \%$ background (blank) reading was set through an area of unstained section adjacent to the measured cell.

Measurements of nuclear and nucleolar diameter were made using a $\times 12.5$ ocular micrometer in combination with the $\times 40$ objective, and volumes were calculated. Measurements of nuclear and nucleolar diameters, nuclear DNA, and cell RNA content were made on a minimum of 30 neurones in every group. Measured neurones in affected groups 
were categorized as to whether nuclear shrinkage had occurred or not, and results are presented for 'normal' and 'shrunken' cells.

\section{RESULTS}

Graphs of nuclear and nucleolar volumes plotted against cytoplasmic RNA, for all neuronal types measured, in both normal and MND cases, are shown in Figs 4-13. Regression lines, gradients (M) and correlation coefficients $(r)$ were calculated. In every case cells with normal nuclei are depicted by an open circle and those with shrunken nuclei by a filled circle.

NUCLEAR VOLUME AND CYTOPLASMIC RNA As an example of a completely unaffected group, we measured Purkinje neurones and found similar linear relationships between nuclear volume and cytoplasmic RNA for the control case $(r=$ $0.56, \mathrm{M}=32)$ and case $\mathrm{A}(r=0.62, \mathrm{M}=29 \cdot 5)$.

Anterior horn cells from the control case also showed a linear $(r=0.62, M=110)$ nuclear volume/cytoplasmic RNA relationship (Fig. 4). By contrast, Fig. 5 demonstrates this relationship, for these cells, in the MND case A; an almost identical picture was seen for case $B$. In these latter two cases, all measured cells of this type had shrunken nuclei. Figure 5 indicates that, as the cell nucleus shrinks, so cytoplasmic RNA is progressively lost from the cell. Initially, there is a large decrease in nuclear volume, accompanied by a low rate of RNA loss, until

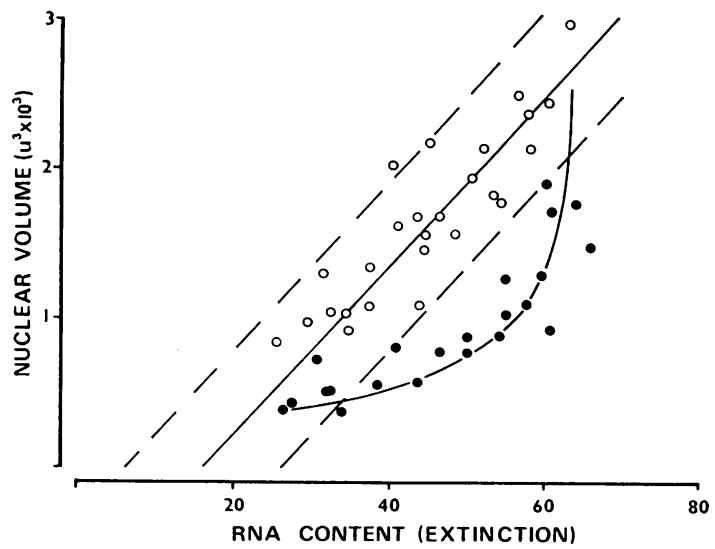

FIG. 6. Graph of nuclear volume plotted against cytoplasmic RNA content for motor cells of the trigeminal nucleus of $M N D$ case $A$.

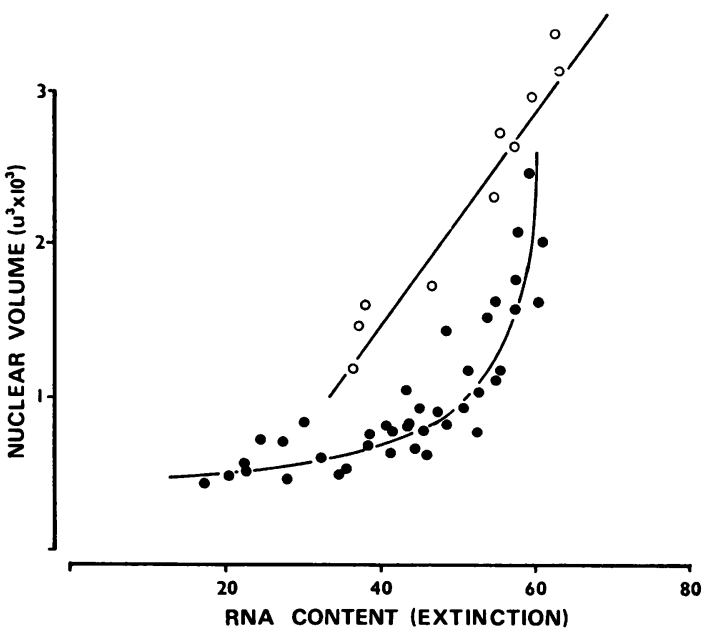

FIG. 7. Graph of nuclear volume plotted against cytoplasmic RNA content for motor cells of the trigeminal nucleus of MND case $B$.

a volume of $1,000 \mu \mathrm{m}^{3}$ is reached when furthe nuclear shrinkage proceeds with proportionatê仓 을 loss of RNA until a minimum volume $\left(250 \mu \mathrm{m}^{3} \vec{D}\right.$ is attained, when RNA loss proceeds without fur- 0 ther nuclear change.

Neurones of the trigeminal motor nucleus from the control patient again show the linear relationship $(r=0.63, M=90)$ between nuclear volume and cytoplasmic RNA similar to that seen for anterior horn cells (Fig. 4). In case A (Fig. 6) and case B (Fig. 7), the values of nuclear

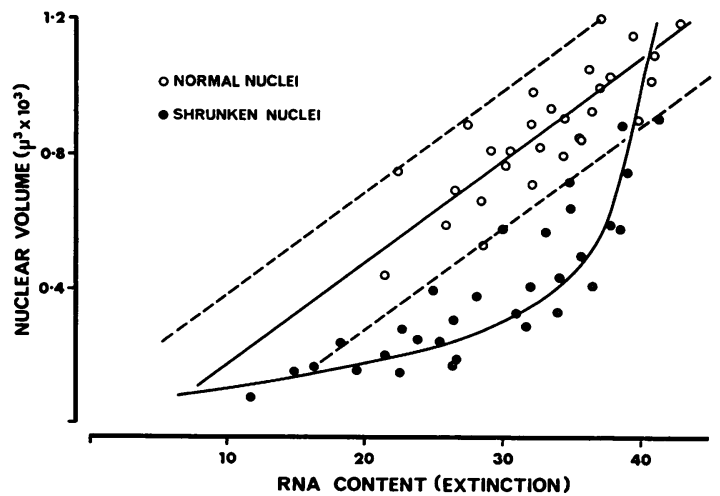

FIG. 8. Graph of nuclear volume plotted against cytoplasmic RNA content for motor cells of the oculomotor nucleus of MND case $B$. 


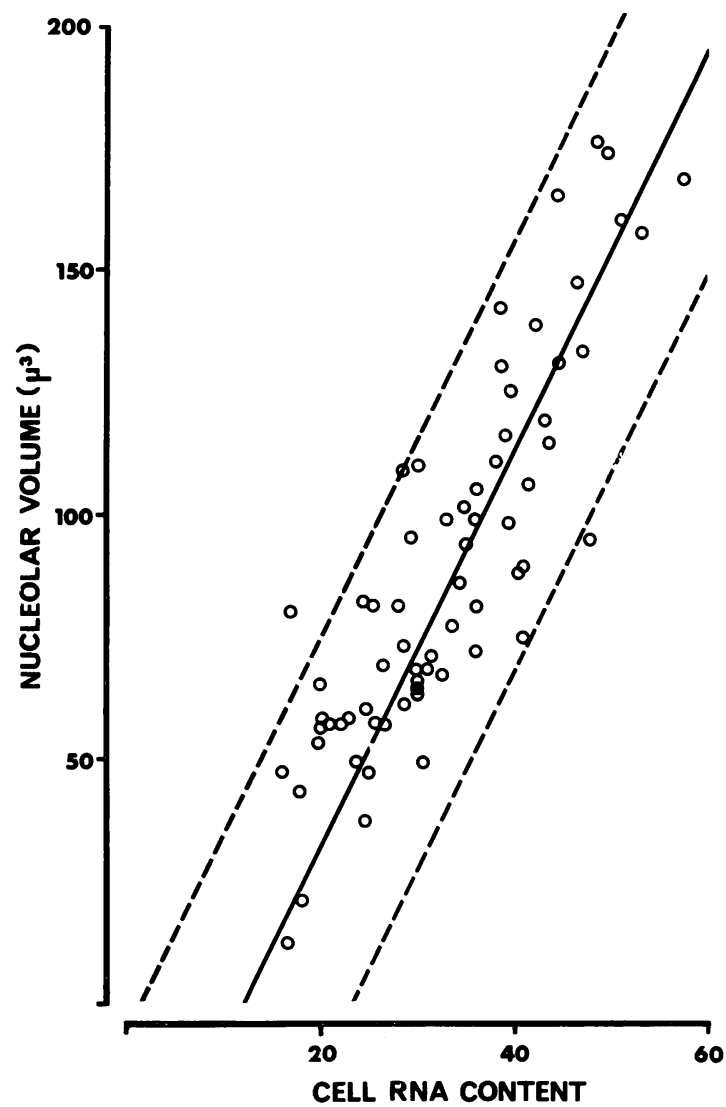

FIG. 9. Graph of nucleolar volume plotted against cytoplasmic (cell) RNA content for anterior horn cells of the control case.

volume and cytoplasmic RNA of 'normal' cells with normal nuclei and 'diseased' cells with shrunken nuclei from the trigeminal group are identified separately. The 'normal' neurones display a nuclear volume/cytoplasmic RNA relationship similar to those of the control patientthat is, $r=0.66, \mathrm{M}=76.7$ for case $\mathrm{A} ; r=0.73$, $\mathrm{M}=74.8$ for case B). However, 'diseased' neurones show a very different picture; a comparison of 'diseased' and 'normal' cells, of equivalent cytoplasmic RNA content, shows a greatly decreased nuclear volume in the 'diseased' neurone relative to that in the 'normal' neurone. This pattern of changes is also present in cells of the oculomotor nucleus of case B (Fig. 8).

It was notable that in the long-standing MND case (B) the proportion of cells showing shrunken nuclei, in a less severely affected nerve nucleus, such as the trigeminal motor nucleus, is greater than that of the accidentally terminated case (A), whereas in severely affected cell groups (anterior horn cells) both cases show that all cells contain shrunken nuclei.

These results indicate, therefore, that neurones affected by the disease initially show a decrease in nuclear volume before cytoplasmic RNA loss.

NUCLEOLAR VOLUME AND CYTOPLASMIC RNA In a manner similar to that used for nuclear volume a linear relationship between nucleolar volume and cytoplasmic RNA was seen both in Purkinje cells of the control case $(r=0 \cdot 81, M=22)$ and of case $\mathrm{A}(r=0 \cdot 78, \mathrm{M}=26)$.

Anterior horn cells of the control case also showed the usual linear nucleolar volume/cytoplasmic RNA relationship $(r=0.64, M=42 \cdot 5)$ (Fig. 9), whereas Fig. 10 shows this relationship for these cells in MND case A. The same picture is seen in case B. As the nucleolar volume and,

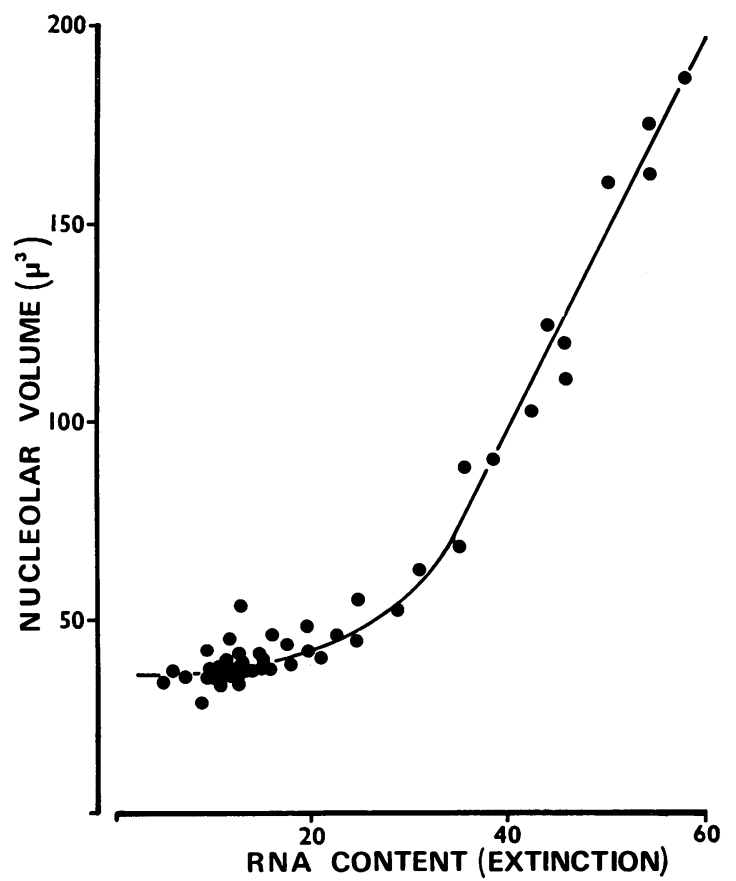

FIG. 10. Graph of nucleolar volume plotted against cytoplasmic RNA content for anterior horn cells of MND case $A$. 


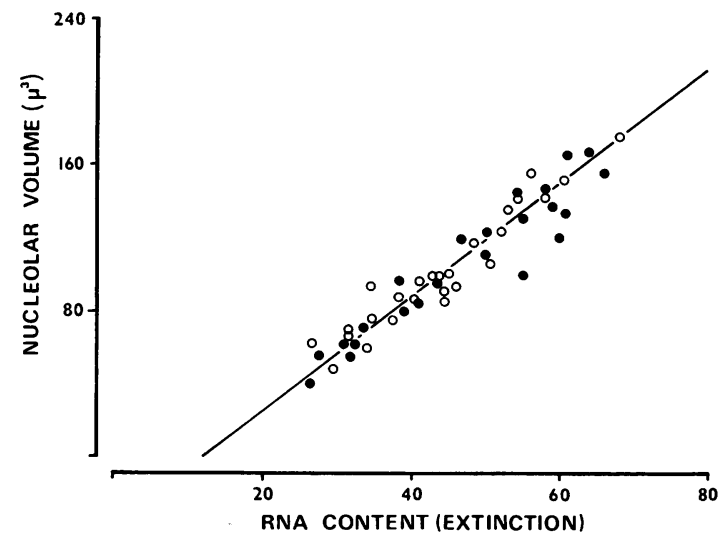

FIG. 11. Graph of nucleolar volume plotted against cytoplasmic RNA content for motor cells of the trigeminal nucleus of $M N D$ case $A$.

hence, nucleolar RNA is reduced, cytoplasmic RNA is lost proportionally until a minimum nucleolar volume of $30 \mu \mathrm{m}^{3}$ is reached when cytoplasmic RNA loss proceeds without further nucleolar shrinkage.

The nucleolar volume/cytoplasmic RNA relationship for cells of the trigeminal motor nucleus of the control case again shows a simple linear slope $(r=0.74, M=34.5)$ as in Fig. 9, whereas Figs 11 and 12 show this relationship for such cells of MND cases A and B respectively. In Figs 11 and 12 the nucleolar volume/cytoplasmic relationship for 'normal' neurones follows a similar course $(r=0.83, M=31)$ for case $A$; $r=0.77, M=32.5$ for case $B$ ) to that of the control case. However, the 'diseased' neuronesthat is, cells with shrunken nuclei-may at first show a normal nucleolar volume with a normal cytoplasmic RNA $(r=0 \cdot 76, \mathrm{M}=30 \cdot 5$ for case $\mathrm{A}$; $r=0.80, M=33$ for case $B)$ but later may show a decrease in nucleolar volume with proportional loss of cytoplasmic RNA until a minimum nucleolar volume of $60 \mu^{3}$ is reached, when cytoplasmic RNA loss proceeds without further nucleolar shrinkage. Similar variation from the control relationship is seen in neurones of the oculomotor nucleus in MND case B (Fig. 13).

NUCLEAR DNA Results of measurements of nuclear DNA content of neurones and glia of the anterior horns of spinal cord, the motor nuclei of the trigeminal and oculomotor nerves and
TABLE

MEAN DNA CONTENT (ARBITRARY UNITS \pm 2 SD) OFO NEURONES AND GLIA OF MND (A AND B) AND CONTROL (C) CASES AS MEASURED BY TWO WAVELENGTH CYTOPHOTOGO

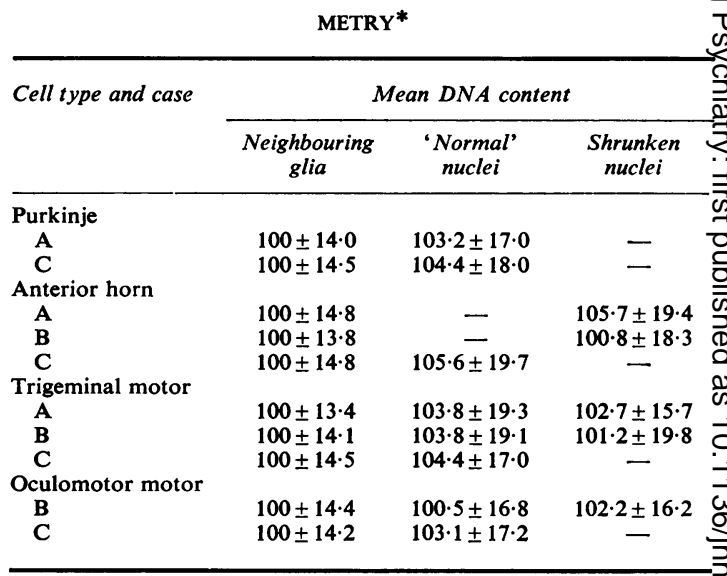

* Because of variations in the nuclear staining intensity of cells from different areas, both between and within cases, which depend on length of fixation and its effect on hydrolysis, measurements of ghiaco cell DNA are calibrated to an arbitrary baseline value of 100 . Nerve-. cell DNA content is scaled accordingly.

cerebellum are shown in the Table. From these results there is no significant difference between the mean DNA content of (1) motor neurongs and glia of all nerve cell groups in the MN要 cases; (2) motor neurones with shrunken a후은 normal nuclei from diseased and normal cefts $\vec{C}$ respectively.

There is, however, a marked difference in the

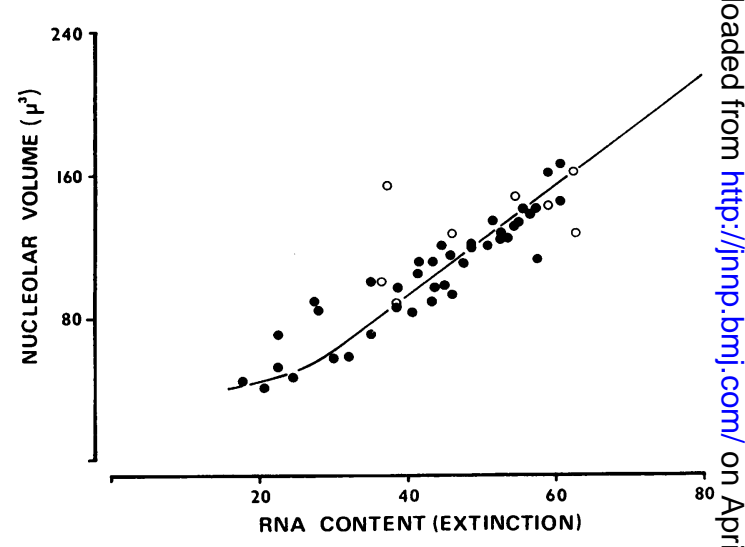

FIG. 12. Graph of nucleolar volume plotted against cytoplasmic RNA content for motor cells of the tri-G geminal nucleus of $M N D$ case $B$. 


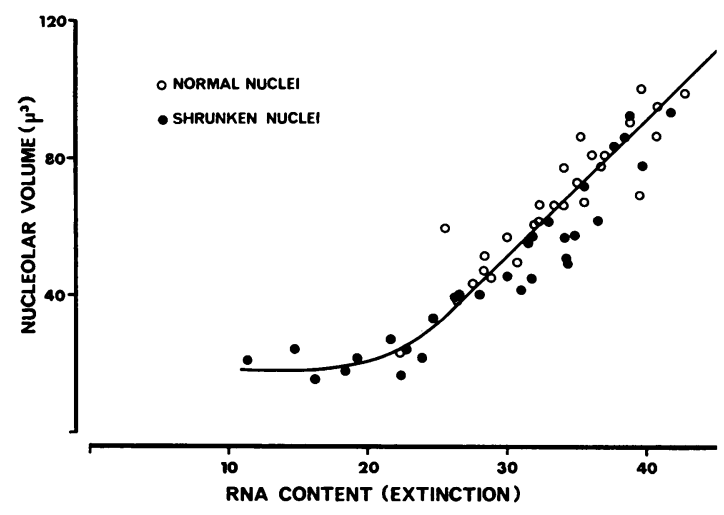

FIG. 13. Graph of nucleolar volume plotted against cytoplasmic RNA content for motor cells of the oculomotor nucleus of MND case B.

appearance of the nuclei; in 'normal' neurones the DNA is finely dispersed throughout the nucleus but in 'diseased' neurones the nucleus is shrunken and the DNA densely stained in heterochromatic granules (Fig. 14).

\section{DISCUSSION}

Measurements of nuclear and nucleolar volume, cytoplasmic RNA, and nuclear DNA contents, clearly show:

1. There is no observed difference between the nuclear and nucleolar volume/cytoplasmic RNA relationships for Purkinje cells of MND and the control cases. As shown by these parameters such cells are considered to be unaffected by the disease process.

2. There is a marked difference from the control relationships for anterior horn cells of both the long-standing and the accidentally terminated MND cases, showing that in both cases these cell types are rapidly and totally affected by the disease process.

3. Those neurones of the trigeminal and oculomotor nerve nuclei of the MND cases which had normal sized nuclei had similar nuclear and nucleolar volume/cytoplasmic RNA relationships to those neurones of the control case. It is valid therefore to consider such cells as normal-that is, unaffected by MND pathogen.

FIG. 14. Motor cell of oculomotor nucleus of MND case B showing nuclear shrinkage with the $D N A$ densely stained in heterochromatic granules. Feulgen, $\times 1,700$. 
4. At least half the neurones of trigeminal and oculomotor nuclei showed early degenerative change in case $\mathrm{A}$ and over $80 \%$ in case $\mathrm{B}$. It is in a study of these two nerve nuclei and a comparison of their 'normal' and 'shrunken' cells that the initial changes due to MND pathogen can be seen.

It appears that the initial site of action of the MND pathogen is the nucleus and its prime effect is to cause (either directly or indirectly) a decrease in nuclear volume accompanied by a condensation of the chromatin nucleoprotein, but without a concomitant loss of DNA, and, furthermore, without effect on the cytoplasmic RNA content. The DNA is presumably inactivated, being changed structurally from a metabolically available diffuse form into a relatively inactive dense form with a consequent reduction in messenger RNA synthesis (Hsu, 1962; Littau et al., 1964). This reduction in nuclear volume (DNA condensation) proceeds with little effect on the cell's metabolic capacity, as indicated by a normally active nucleolus and normal cytoplasmic ribosomal RNA content, until a volume of $1,000 \mu \mathrm{m}^{3}$ is reached. At this point, the nucleolus shrinks and cytoplasmic RNA begins to be lost from the cell.

Since cell protein synthesis is dependent upon the rapid turnover of a small pool of messenger and transfer RNA molecules (Koenig, 1968b) derived from the nucleus, once supplies of these are reduced cell synthetic activity will soon diminish. This will result in a failure to supply the cell with the essential protein (enzymes) necessary to maintain its metabolic economy. Furthermore, the nucleolus is seen to decrease both in volume and basophilia (a sign of decreased ribosomal RNA output), with a parallel loss of cytoplasmic RNA and a decrease of protein synthetic capacity within the cell. The remaining RNA (Nissl) granules become fused into conglomerate masses before cell death and eventual removal by lytic activity.

These changes are slowly progressive and the neurological syndrome is brought about by the dysfunction of at first isolated and later increasing numbers of neurones, until involvement of affected cell types is total. During this time, diseased neurones may remain capable of function (even if abnormally) until they finally succumb to the MND pathogen.
As a result of studies mainly by Koenig and co-workers, an experimental myelopathy has ồ been induced in cats using certain metabolic $\stackrel{\text { ? }}{=}$ inhibitors, which shows many of the features 0 typically seen in MND. Such neurotoxins are the fluoropyrimidines, 5-fluoro-orotic acid (FO) and 5-fluoro-uridine (FUR) and the antibiotics actinomycin D (AMD) and related compounds. Both sets of compounds interfere with protein $\overrightarrow{\vec{c}}$ synthesis, although the nature of the inhibition is different in each case:

FO is incorporated into messenger $(\mathrm{m})$, ribosomal (r), and transfer (t) RNA forms as 5- $\mathbb{\mathbb { R }}$ fluorouridine 5'monophosphate (Koenig, 1967) and inhibits protein synthesis by (1) the quantitative reduction of all RNA species; (2) the formation of fluorouracil substituted RNA species; (3) the reduction in number of polyribosomes, the seat of protein synthesis, while monoribosomes increase relatively.

AMD is a potent inhibitor of DNA directe m RNA synthesis by binding onto the DNA tem- $\overrightarrow{0}$ plate and interfering with the function of RNS polymerase (Reich and Goldberg, 1964). Unlik FO, AMD blocks the transcription of the DN template without affecting pyrimidine synthesisg $\mathbb{D}$ and, because of its highly selective action, offers $\frac{\mathbb{O}}{1}$ a unique opportunity for correlating neurog 3 biological activity with RNA synthesis.

When these compounds were injected intra thecally into the lumbar spinal cord, after a latent period a variety of neurological disturbances appeared which progressed until death. After three to four days muscle twitches and myoclonic jerks were seen (Young et al., 1964) and the hind limbs became clumsy and weak. The flexor muscles were hypertonic and stretch reflexes were seen. Muscle fasciculations due to hyperirritable motor neurones were common. A spastic weakness developed into an areflexic paralysis. The forelimbs might be later affected before the animal succumbed to respiratory paralysis.

With AMD the earliest discernible pathological change was the formation of clumps of dense chromatin in the nucleoplasm of neurones and glia (Koenig, 1966; Koenig and Jacobson, 1966, 1967; Koenig et al., 1967) which was coincident with a blocking of protein synthesis. These nuclear changes, when advanced, were particularly conspicuous in the large motor 
neurones in which normally the bulk of the chromatin was diffuse and only a few dense granules were seen. Nucleolar alterations followed chromatin condensation with loss of basophilia, shrinkage, and vacuolization. Cytoplasmic RNA (Nissl) was unaffected until 24 hours after injection, when chromatolysis occurred and spread throughout the cell body. There was also seen an early loss of acetylcholinesterase (AchE) and thiamine pyrophosphatase (TPPase) activities with a disruption of the Golgi network (Koenig, 1968b). There was, however, a relative persistence of and increase in acid phosphatase activity, while loss of oxidative enzymes was seen later.

These results suggest that AMD exerts its biological action through an alteration of the structure of the DNA template by inducing the condensation of the diffuse chromatin. Koenig (1968b) postulated that AMD, on binding to DNA, cross-linked the metabolically active microfibrils of diffuse chromatin, converting them into the contracted, relatively impervious, dense chromatin mass. This physical state would be sufficient to account for the inactivity of such chromatin as a template in the RNA polymerase catalysed synthesis of RNA (Koenig et al., 1967).

The results of our studies on human MND and those of Koenig's experimental myelopathy, taken together, therefore present strong evidence for the view that a MND pathogen causes a slowly advancing condensation of the DNA leading to a progressive restriction in the rate of transcription of $\mathrm{m}, \mathrm{r}$, and $\mathrm{t}$ RNA, either together or individually. The ensuing curtailment of RNA and protein synthesis initially has little effect on survival of the neurone as demonstrated by the occurrence of pyknotic nuclei in otherwise normal cells which show normal nucleoli and no cytoplasmic RNA loss. However, as chromatin condensation proceeds, increasingly severe restrictions are imposed upon the m RNA output leading to neuronal dysfunction and loss of cytoplasmic and nucleolar RNA. Eventually, the nucleus is unable to supply the cell with the necessary amounts of m RNA and cell function ceases, and those atrophic changes which eventually lead to cell death commence.

We wish to thank the National Fund for Research into Crippling Diseases which generously supported this work.

\section{REFERENCES}

Cavanagh, J. B. (1968). Organo-phosphorus neurotoxicity and the 'dying back' process. In Motor Neuron Diseases, pp. 292-300. Edited by F. H. Norris, Jr, and L. T. Kurland. Grune and Stratton: New York.

Charcot, J. M., and Joffroy, A. (1869). Deux cas d'atrophie musculaire progressive, avec lésions de la substance grise et des faisceaux antéro-latéraux de la moelle épinière. Archives de Physiologie, Normale et Pathologique, 2, 354367, 744-760.

Engel, W. K. (1968). Motor neuron histochemistry in ALS and infantile spinal muscular atrophy. In Motor Neuron Diseases, pp. 218-234. Edited by F. H. Norris, Jr, and L. T. Kurland. Grune and Stratton: New York.

Friede, R. L. (1968). Enzyme histochemical changes in $A L S$. In Motor Neurone Diseases, pp. 235-241. Edited by F. H. Norris, Jr, and L. T. Kurland. Grune and Stratton: New York.

Friede, R. L., and DeJong, R. N. (1964). Neuronal enzymatic failure in Creutzfeldt-Jakob disease. Archives of Neurology (Chic.), 10, 181-195.

Gibbs, C. J., Jr, and Gajdusek, D. C. (1968). Kuru-a prototype subacute infectious disease of the nervous system as a model for the study of amyotrophic lateral sclerosis. In Motor Neuron Diseases, pp. 269-279. Edited by F. H. Norris, Jr, and L. T. Kurland. Grune and Stratton: New York.

Hsu, T. C. (1962). Differential rate in RNA synthesis between euchromatin and heterochromatin. Experimental Cell Research, 7, 332-334.

Koenig, H. (1966). Cytochemical effects of actinomycin D in cat spinal cord. (Abstract.) Journal of Histochemistry and Cytochemistry, 14, 814-815.

Koenig, H. (1967). Neurobiological action of some pyrimidine analogs. International Revue of Neurobiology, 10, 199230.

Koenig, H. (1968a). Histochemical clues to metabolic abnormalities in nerve cells. In Motor Neuron Diseases pp. 245-249. Edited by F. H. Norris, Jr and L. T. Kurland. Grune and Stratton: New York.

Koenig, H. (1968b). Neurobiologic effects of agents which alter nucleic acid metabolism. In Motor Neuron Diseases, pp. 347-368. Edited by F. H. Norris, Jr and L. T. Kurland. Grune and Stratton: New York.

Koenig, H., and Jacobson, S. (1966). Nuclear changes induced in neurons by actinomycin D. (Abstract.) Journal of Cell Biology, 31, 61 A-62A.

Koenig, H., and Jacobson, S. (1967). Cytochemical and ultrastructural effects of actinomycin $D$ in cat spinal cord. (Abstract.) Journal of Neuropathology and Experimental Neurology, 26, 148-149.

Koenig, H., Lu, C., and Jacobson, S. (1967). Effect of actinomycin D (AMD) and related antibodies on biosynthetic activities and chromatin morphology in cat spinal cord. (Abstract.) Federation Proceedings, 26, 291.

Kurland, L. T., and Mulder, D. W. (1954). Epidemiologic investigations of amyotrophic lateral sclerosis. 1. Neurology (Minneap.), 4, 355-378, 438-448.

Kurland, L. T., and Mulder, D. W. (1955). Epidemiologic investigations of amyotrophic lateral sclerosis. 2. Neurology (Minneap.), 5, 182-196, 249-268.

Littau, V. C., Allfrey, V. G., Frenster, J. H., and Mirsky, A. E. (1964). Active and inactive regions of nuclear chromatin as revealed by electron microscope auto- 
radiography. Proceedings of the National Academy of Sciences of the United States of America, 52, 93-100.

Mann, D. M. A. (1972). Unpublished results.

Mendelsohn, M. L. (1958). The two-wavelength method of microspectrophotometry. 2. A set of tables to facilitate the calculations. Journal of Biophysiology and Biochemical Cytology, 4, 415-424.

Ornstein, L. (1952). The distributional error in microspectrophotometry. Laboratory Investigation, 1, 250-265.

Patau, K. (1952). Absorption microphotometry of irregularshaped objects. Chromosoma, 5, 341-362.

Reich, E., and Goldberg, I. H. (1964). Actinomycin and nucleic acid function. In Progress in Nucleic Acid Research and Molecular Biology, 3, 183-234.
Robinson, N. (1966). A histochemical study of motब्ष neurone disease. Acta Neuropathologica, 7, 101-110.

Shea, J. R., Jr (1970). A method for in situ cytophotometre estimation of absolute amount of ribonucleic acid usiff azure B. Journal of Histochemistry and Cytochemistry, 180 143-152.

Young, I. J., Dabbouseh, J., and Koenig, H. (1964). Effects $\stackrel{\rho}{\mathcal{Q}}$ the pyrimidine analogue 5 -fluorouridine on the cat centro nervous system. (Abstract.) Neurology (Minneap.), 14, 26

Zil'ber, L. A., Bajdakova, Z. L., Gardaš'jan, A. N., Konovalo N. V., Bunina, T. L., and Barabadze, E. M. (1963). AP study of the etiology of amyotrophic lateral sclerosis Bulletin of the World Health Organization, 29, 449-456. 